\title{
115 VARIATIONS IN HOSPITAL DEATH
}

10.1136/bmjspcare-2011-000053.115

A Osinowo, A Pring, J Verne South West Public Health Observatory, Bristol, UK

Background Death largely occurs in old age and there are large variations in the proportion of hospital deaths by Local Authorities (LAs) in England. In the period between 2007 and 2009 deaths occurring in hospital ranged between $43 \%$ and $75 \%$ with about $12 \%$ of LAs having less than $50 \%$ hospital deaths. Funnel plots are powerful tools that can be adapted for use in showing and understanding the variations in hospital deaths in English LAs.

Aim To explore variations in proportion of hospital deaths in LAs by age of population.

Methods Age specific mortality data relating to place of death were extracted from ONS mortality files for years 2007-2009. Hospital deaths as a proportion of all deaths in LAs were calculated and funnel plots were used to study variations by population size and by age.

Results The proportions of LA deaths in hospital are overdispersed, with more LAs lying outside the control limits of the funnel plot than would be expected if the size of a LA were the only factor predicting hospital death. Factors such as social deprivation, demographics and local End of Life (EOL) care service configuration contribute to the overdispersion for example, some LAs provide specialist palliative care services within hospitals while others provide this service elsewhere. EOL care in less well organised LAs may result in people near the EOL being unnecessarily admitted to die in hospital.

Conclusions Place to place comparison of hospital deaths reveals stack differences which must be understood to ensure equality of care. 